\title{
Exploring genetic variation based on drought- induced phenotypic alterations during reproductive stages in Desi and Kabuli types of chickpea
}

\author{
Alireza Taleei ( $\nabla$ ataleei@ut.ac.ir) \\ University of Tehran https://orcid.org/0000-0001-7143-6694 \\ Jalal Shaabani \\ University of Tehran
}

\section{Research article}

Keywords: path analysis, yield potential, variance components, genotypic effects, yield stability

Posted Date: January 22nd, 2020

DOI: https://doi.org/10.21203/rs.2.21488/v1

License: (c) (i) This work is licensed under a Creative Commons Attribution 4.0 International License.

Read Full License 


\section{Abstract}

Background: Identification of appropriate varieties adapted to the global warming and enhanced drought changes is imperative. In this study two sets of thirty elite genotypes of Desi and Kabuli chickpea types were used to investigate the effects of water scarcity at the phenotypic level in reproductive stages and surveying different drought-induced responses for them.

Results: Alterations in GY and its components, FT, PT, MT, FM, and SDM as well as number of drought tolerance indices were measured in field conditions. The estimated genotypic effects were detected significant at both limited and full irrigation conditions for GY, GN, GW, and SDM; however, these effects had smaller values for environmental effects except in GW. The SDM and GW in water-limited conditions showed significant positive relationship with those of full irrigated for both chickpea types. GMP index provided the most positive correlations with GY for both type either of two conditions. The highest direct effect on GY was represented by SDM for Kabuli at both conditions as well as Desi chickpeas in limited water condition, while GN was the most one in full-irrigated Desi chickpeas. The ideal genotypes, 25 and 321 , as Kabuli and Desi chickpeas, respectively, were detected with high stable and high GY.

Conclusions: Results of this study showed that tested chickpea genotypes responded differently under different water treatments, suggesting the importance of assessment of genotypes under these conditions in order to identify the best genotype make up for each particular condition. As water stress severity was applied equally, therefore it was thought to be more serious in genotypes with a greater life cycle. However, it seems that chickpea plants have been adapted to the terminal drought stress, which could be due to the same time of vegetative growth with filling pods and transfer capability of photosynthesis assimilates towards more grain yield in tolerant genotypes. It seems to change in plant phenology due to the terminal drought stress more affected GN and GW in Desi and Kabuli chickpeas, respectively. These differences could be clear points for the leadership of breeding programs towards more adaptation of both Desi and Kabuli chickpea types to terminal water stress, respectively. Moderate to a high proportion of $\mathrm{G} \times \mathrm{E}$ effects were observed in combined analysis for GY, GN, and SDM compared to genotypic effects, suggesting that $\mathrm{G} \times \mathrm{E}$ effects played a greater role than genotypic effects. The ideal genotype of Kabuli type i.e. genotype 25 had greater GY as well as SDM in water-limited condition, while genotype 321 as ideal Desi genotype showed acceptable GY and SDM, but could compensate with higher GN.

\section{Introduction}

The average of global temperatures has shown an increase of $1.2^{\circ} \mathrm{C}$ over the past century and it is estimated to rise up to $3{ }^{\circ} \mathrm{C}$ for 2100 because of global warming [28]. So is widely predicted to increase the frequency and intensity of drought, accompanied by the higher temperatures and higher $\mathrm{CO} 2$ concentration in semi-arid and sub-tropical regions out of the climate changes [37]. These alterations 
could lead to an increase in the rate of evaporation and decrease water availability for crop plants and resulting in yield losses, potentially as a threat to human food security. Thus, multiple improvement strategies are necessary for sustainable crop production, especially since the production of major crop plants could be reduced over $50 \%$ under drought stress conditions [9].

Chickpeas with black or brown-colored grain coat are categorized by Desi type and with creamy or beigecolored are named Kabuli. The Desi type is more prominent by accounts up to $80 \%$ globally and has a more small grain size as well as thicker grain coat than Kabuli. Chickpea grain provides necessary amino acids (lysine, methionine, threonine, valine, isoleucine, and leucine) to the human diet and health because of up to $70 \%$ carbohydrates, $10 \%$ fat, $23 \%$ fiber, and $31 \%$ protein - higher than any other pulse crop. That of the root, in addition, enriches soil fertility by atmospheric nitrogen fixation $[33,16]$

As a cool-season grain legume, chickpea is predominantly grown in semi-arid regions and its reproductive stages are usually faced with the lack of rain [32]. These regions globally are classified in two major types, i.e. stored soil moisture in subtropical environments with summer-dominant rainfall and rainfall in winter-dominant Mediterranean-type environments that suffer yield losses due to terminal drought stress as rain-fed farming systems of chickpeas growing conditions [40,34]. Nevertheless, water scarcity is an important scourge, which can be addressed by three aspects including severity, timing, and duration. Iran, the fourth major global chickpea producer after India, Pakistan, and Turkey, is a region with the Mediterranean precipitation and has been composed of arid and semi-arid lands with about $250 \mathrm{~mm}$ of annual rainfall, equivalent to one-third of average rainfall in the world, in which water scarcity arises owing to its shortage at the end of spring. A large number of studies have been represented in chickpea plants under drought stress and without drought stress, which some of them in phenotypic levels of agronomic traits are mentioned. Behboudian et al (2001) presented that although shoot dry matter and GY of a Desi chickpea cultivar "Sona" were decreased by terminal drought stress, it had not to effect on GW [4]. In addition, Pushpavalli et al (2014) found that drought at the reproductive stage of chickpea can reduce GY and GN but could not influence GW [22]. Kabuli chickpeas with early flowering and early maturity as well as with high $\mathrm{HI}$ were maintained as ones, which presented more GY under terminal drought stress [26]. In a comparative study, Kabuli chickpeas have shown a fewer reduction in SDM, while more decreased in GY, GN, HI, and GW have been shown than Desi under terminal drought stress [20]. GY and $\mathrm{GW}$ reduced by $53 \%$ and $4.5 \%$, respectively, and MT was earlier 2 days for chickpeas grown under the dry-land condition as compared to irrigated condition [36]. Path analysis on chickpea plants has showed that GN per plant and GW have most direct effect contributions for GY under non-drought stress condition $[14,27]$, while SDM and $\mathrm{HI}$ were those of in drought stress condition [2, 21].

Drought tolerance selection has been made as a complicated procedure because of the interactions between genotypes and environments, causing restricted knowledge about the role of tolerance mechanisms. Drought can cause yield losses if plants do not get enough water during the reproductive stage especially in seed filling, which has been a common scenario that Mediterranean lands chickpea farmers face. Unfortunately, knowledge of suitable plant attributes to screen large numbers of genotypes with the aim of improving crop performance in water scarcity, which could be obtained in a short time 
and be cost-effective remains a major challenge in plant breeding. Nevertheless, drought response could explain genotypic variation for terminal drought tolerance in Desi and Kabuli chickpeas. To investigate this hypothesis, we have used a phenotyping study on Kabuli and Desi chickpea types to examine their responses under terminal water-limited and full irrigation conditions, with the aim of exploring the genetic variations of tolerant genotypes and the state of relationships among plant traits.

\section{Methods}

\section{Field location and experimental materials}

The field experiment was conducted in the research field of the Department of Agronomy and Plant Breeding, the University of Tehran located in Karaj, Iran ( $35^{\circ} 56^{\prime} \mathrm{N}, 50^{\circ} 58^{\prime} \mathrm{E}, 1112.5 \mathrm{~m}$ a.s.I.). Experimental plant materials consisted of 28 Desi and 28 Kabuli elite chickpea genotypes with two Iranian chickpea cultivars as check by named Pyrooz and Kaka in Desi type as well as Jam and Koorosh in Kabuli type obtained from the Departmental Gene Bank of the University of Tehran [See 30 and 31 for more details].

\section{Experimental Design implementation and data collection}

The experiment was laid out in a randomized complete block Design with two replications. Each plot included 1-meter single row by $50 \mathrm{~cm}$ distances and $10 \mathrm{~cm}$ plant-to-plant spacing. Blocks were considered as environments, where the genotypes were randomly distributed in each of those, that the experiment had two environments include water-limited and full irrigation conditions. The cultivation was carried out in the period of February to August 2014, and differential in plant furrow irrigation was started at $50 \%$ of the flowering stage, which stopped in the water-limited conditions (rain-fed condition), while continued through crop maturity followed a common irrigation regime of the region in the full water irrigation conditions. The measured phenological traits included days to $50 \%$ of flowering, days to $50 \%$ of podding, days from flowering to crop maturity, and days to maturity. Eight number of plants, excluding border plants, were harvested individually after the maturity to measure of the shoot dry matter (g plant1), grain yield (g plant-1), 100-grain weight (g plant-1), and a number of grains (plant-1) under both conditions. Harvest index (plant-1) was calculated from the formula: $\mathrm{HI}=$ grain weight/shoot dry matter. These harvested plants left out for shade drying about a week in separate paper bags before weighting. Drought tolerance indices were calculated based on grain yield in full irrigation and limited water irrigation treatments, represented in Table 1. The degree of stress intensity (SI) applied on plants under water-limited conditions achieved using the formula: $\mathrm{SI}=(1-(\mathrm{Yp} / \mathrm{Ys}))$, where, YP is the mean grain yields in full irrigated treatment and YS is the mean grain yields under limited water irrigation treatment.

\section{Statistical model and data analysis}

A visualized analysis of genotype main effect and genotype by environment interaction effects (GGE) was worked using GGEbiplot software version 6.0 to evaluate the yield stability in interaction by an environment of the tested chickpea genotypes followed Yan et al (200). Path analysis, a standardized partial regression coefficient was conducted to decompose correlation coefficients into components of 
direct and indirect effects as well as to examine the strength of the contribution of the various measured traits on grain yield. This purpose was followed using SmartPLS software (version 3.0, SmartPLS GmbH, Boenningstedt, Germany) with partial least square structural equation modeling (PLS-SEM) method developed by Wold (1982). To estimate variance components, minimum norm quadratic unbiased estimation (MINQUE) as a linear mixed model approach (Rao, 1971, Zhu, 189) was deployed in R software version 3.5.1 (R Development Core Team, 2018; www.R-project.org). As deviations from the population mean, in addition, genotypic effects in limited water conditions and full irrigation conditions as well as in a combined analysis were predicted separately by adjusted unbiased prediction method (Zhu, 1993). A significant test of interesting parameters (components of variance and genetic effects) was done according to a randomized 10-group Jackknife method to estimate standard errors (Wu, 2012). An R package named MINQUE performed the estimation of components of variance and prediction of genotypic effects by $\mathrm{Wu}$ (2012). These estimations were calculated using a linear mixed model for environmental, chickpea type, genotype, and the interaction of genotype by environment effects followed by equation (1):

$$
\text { (1) } Y_{i j k}=\mu+E_{i}+G_{j}+T_{k}+\mathrm{GE}_{i j}+B_{l(i)}+e_{i j k}
$$

where $Y i j k$ is an observation; $\mu$ is a population mean; $E_{i}$ is an environmental effect; $T_{k}$ is a type of chickpea effect; $G_{j}$ is a genotypic effect; $\mathrm{GE}_{i j}$ is a genotype by environment interaction effects; $B_{I(i)}$ is a block effects within environment; and $e_{i j k}$ is a random error.

In addition, each environments was analyzed independently in a completely randomized block Design with the linear mixed model followed by equation (2):

$$
\text { (2) } Y_{i j}=\mu+B_{i}+G_{j}+e_{i j}
$$

where $Y_{i j}$ is an observation; $\mu$ is a population mean; $B_{i}$ is a block effect; $G_{j}$ is a genotypic effect; and $e_{i j}$ is a random error.

The degree of overall linear association for each of the traits between water-limited and full irrigation conditions was estimated by scattering charts in Microsoft Excel 2013 (Microsoft Corporation, Redmond, WA, USA). Furthermore, Pearson's correlation coefficients determined the degree of associations between the drought tolerance indices and phenotypic measured plant characters via IBM SPSS Statistics (version 22.0, SPSS Inc, Chicago, IL).

\section{Results}

\section{Variance components}

Estimated variance components, which expressed as the proportions of the phenotypic variances for limited and full irrigation conditions are summarized in Tables 5 and 6, respectively. The estimated genotypic effect variances were detected highly significant $(P \leq 0.001)$ for $G Y, G N, G W$, and SDM in 
Kabuli chickpea genotypes under water-limited treatment. Nevertheless, there were highly significant ( $\mathrm{P} \leq$ 0.001) effects of genotypic variances in those of GN, GW, and SDM as well as a significant effect (P $\leq$ $0.05)$ for $G Y$ in Desi chickpea genotypes at the limited water condition. A moderate significant $(P \leq 0.01)$ effect of genotypic variance was observed for GY in full irrigation treatment; also, the genotypic variances had highly significant $(P \leq 0.001)$ effects for $G N, G W$, and SDM in Kabuli chickpeas. The Desi type of chickpea genotypes showed highly significant effects $(P \leq 0.001)$ for variances genotypic effects for all the aforementioned traits in full irrigation treatment. The estimated variances of genotypic effects for both chickpea types were larger than residual effects except for that of GY in limited and full irrigation conditions. In a combined model analysis, the estimated environmental variance had the largest values for $G Y, G N$, and SDM with highly significant effects $(P \leq 0.001)$. The estimated variances for chickpea types have highly significant effects $(P \leq 0.001)$ which were observed for $G Y, G W$, and SDM, respectively. The estimated genotypic variances for $G N, G W$, and SDM were highly significant $(P \leq 0.001)$.

Nevertheless, highly significant effects $(P \leq 0.001)$ for $G Y, G N$, and SDM as well as moderate significant effect $(P \leq 0.01)$ for $G W$ were estimated as genotype by environment variances represented in Table 7 .

\section{Predicted genotypic effects}

The predicted genotypic effects of chickpea genotypes tested under limited water and full irrigation treatments for GY, GN, GW, and SDM are given in Tables 8 and 9, respectively. Among Kabuli type chickpeas, four genotypes $(25,21,166$, and 15$)$ presented Desirable positive significantly genotypic effects for GY under water-limited condition. The positive significant predicted genotypic effects were observed for two Desi chickpea genotypes (321 and 10) in water-limited condition by GY. However, each one of two Kabuli and Desi types had three genotypes with positive significant predicted genotypic effects as Kabuli genotypes 101, 21, and 339 and Desi genotypes 276, 322, and 9 for GY in full-irrigated treatment. The predicted genotypic effects for GY in the combined model analysis were detected significant and positive in five Kabuli genotypes (101, 21, 25, 339, and Jam) and two Desi genotypes (321 and 322) of chickpea presented in Table 10. Two Kabuli (15 and 21) and two Desi (47 and 321) chickpea genotypes showed significantly positive predicted genotypic effects by GN under water-limited condition. In full irrigation treatment, however, only genotype 101 from Kabuli chickpea type detected a positive and significant predicted a genotypic effect on GN. The combined model analysis distinguished the genotypes 21 and 101 from Kabuli type as well as the genotypes 321, 90, 47, 50, and 49 from Desi type as compared to others by a significant positive predicted genotypic effect on GN. The genotype 8 from Desi type was the only one having a positive significantly predicted genotypic effect for GW in limited water treatment. Three genotypes including one Kabuli (15) and two Desi (8 and 9) showed a predicted genotypic effect on GW, positive and significant in full irrigation treatment. These two Desi genotypes i.e. 8 and 9 also were only ones with positive and significant predicted a genotypic effect on GW in combined mode analysis results. For SDM, the predicted genotypic effects were found positive and significant on three Kabuli $(21,25$, and 15) and two Desi (10 and 321) chickpea genotypes at the water-limited condition. Additionally, the genotypes 101 and 21 from Kabuli type and 5 and 322 from Desi type presented for SDM a significant and positive predicted genotypic effect under full irrigation treatment. 
The combined model analysis detected the genotypes 101, 21, 308, and Jam from Kabuli type and the genotype 5 from Desi type as ones showing significant positive predicted genotypic effects on SDM.

\section{Association of GY, GN, GW, and SDM between water-limited and full irrigation conditions}

The correlation analyses performed to examine the dependency of multiple assessed traits of thirty genotypes from each Kabuli and Desi chickpea types in water-limited conditions with its potential in full irrigation condition have been shown in Figure 1. A positive correlation was detected between GY under water-limited treatment and GY in full irrigation treatment for Kabuli type, which was significant at the 0.05 probability level. In the case of $\mathrm{GN}$, the correlation between water-limited and full-irrigated conditions was identified at the 0.01 probability level for Desi type. GW had a significant $(P \leq 0.01)$ and positive correlation between limited water and full-irrigated conditions in both Kabuli and Desi types. These correlations were detected in Kabuli type at 0.05 and in Desi type at 0.01 significantly levels for SDM of chickpea plants.

\section{Pearson's correlation analysis of phenotypic traits and the drought tolerance indices}

The phenotypic correlations calculated between traits and drought tolerance indices are presented in Tables 3 and 4 for Kabuli and Desi chickpea genotypes, respectively. GMP and HM indices showed significant and positive correlations with GY, GN, and SDM of Desi chickpeas under both of water-limited and full-irrigated treatments. However, in the case of Kabuli chickpeas, GMP and HM showed positive significant correlations with GY, SW, GN, and SDM in both of water-limited and full-irrigated treatments, also with $\mathrm{HI}$ only in full-irrigated ones. SSI showed significant positive and negative correlations with GN, SDM, and GY of Kabuli chickpeas in full irrigation and limited water conditions, respectively. Among Desi chickpeas, positive significant correlations were recorded between SSI and HI, SW, SDM, and GY in fullirrigated treatments, while negative and significant correlations were observed for SSI with GN and GY in limited water condition. MP index correlated significantly and positively with GN, SDM, SW, and GY in Kabuli type at the limited water condition. Furthermore, the Kabuli chickpeas showed positive significant correlations with MP by GY, SW, GN, HI, and SDM in full irrigation treatment. In Desi type also MP index had positive and significant correlations with GY and SDM at the both of conditions as well as with GN and SW only in full irrigation ones. TOL index revealed positive and significant correlations with GN, GY, SDM, and $\mathrm{HI}$ in Kabuli and with HI, SDM, GY, and SW in Desi chickpeas, both of them at the full irrigation conditions. Although in Desi type, a significant but negative correlation was observed between the TOL index and GN in limited water treatment. Positive correlations of GN, GY, and SDM in water-limited and SW in both water-limited and full irrigation treatments with the DI index in Kabuli chickpeas were detected significant. In addition, this DI index in Desi type had a negative significant correlation with $\mathrm{HI}$ in full irrigation condition and showed with GN, SDM, and GY in limited water treatment. GY, SDM, and GN presented positive and significant correlations with SNPI index for both Kabuli and Desi chickpea types in limited water condition. In particular, Kabuli chickpeas showed a positive correlation with SNPI by SW significantly, at both limited and full water irrigation treatments. ATI revealed significant positive correlations in Kabuli type with GY, SDM, GN, and $\mathrm{HI}$ in full irrigation treatment and had negative 
significant correlations with GN, SDM, and GY in limited water conditions. GN and GY of Desi chickpeas showed a negative significant correlation with ATI in limited water treatment, however, the correlations were detected positive and significant between ATI index and GY, SW, and HI at the full-irrigated condition. STI index provided significant and positive correlations with GY, SDM, and GN of Desi chickpea type at both limited and full water treatments. Type of Kabuli traits including GY, GN, SDM, and SW were those of by positive and significant correlations with STI in both treatments, though this correlation was observed through $\mathrm{HI}$ in full irrigation ones. SDM, GN, HI, and GY of produced Kabuli chickpeas in full irrigation treatment showed significant correlations with K1STI, positively. This modified STI index had positive significant correlations with GN, GY, SW, SDM, and $\mathrm{HI}$ in full-irrigated condition as well as with SDM in water-limited condition among Desi type of chickpea genotypes. In Kabuli chickpeas, K2STI was correlated positively and significantly with SDM and SW at both limited and full irrigation treatments but also had the correlations with GN and GY only in water-limited ones. In Desi chickpeas, GN and SDM showed in both irrigation treatments, and GY had in water-limited treatment, significant and positive correlations with the K2STI index.

\section{Yield stability analysis}

An average environment coordination (AEC) method developed by Yan (2001) evaluated the yield stability of chickpea genotypes among both Desi and Kabuli types (Figure 2). This method draws a line through the average environment, which has been highlighted with a blue circle dot on this line by named average environment axis that serves as the abscissa of the AEC. The abscissa line is drawn in one direction toward more yields as well as a larger genotype main effect, crossed from biplot origin. Upright to this line, AEC ordinate line places high yielding genotypes in its right half and those of low yielding has been located in the left half. The visualized yield stability analysis based on principal component analysis represented a biplot as justified $72.14 \%$ and $27.86 \%$ by PC1 (horizontal axis) and PC2 (vertical axis), respectively. The results of yield stability analysis demonstrated that genotype 25 from Kabuli and genotype 321 among Desi chickpeas were the best and ideal ones, which presented high grain yield with high stability based on the AEC analysis. These two ideal genotypes, although, have been located away from the center of concentric circles, showed the least distance from the average environment. In next grade genotypes, 166, 92, and 15 from Kabuli type and genotype 10 from Desi type showed suitable mean yields and stability taken from a distance of concentric circles.

\section{Path analysis}

The strength of the contribution of the different measured traits on grain yield was defined by path diagram analysis as having been shown in Figures 3, 4, 5, and 6. It was observed for Kabuli chickpeas that $\mathrm{HI}, \mathrm{GN}$, and SDM had a direct positive contribution of $0.302,0.153$, and 0.722 , respectively, on grain yield in full irrigation condition. However, in water-limited treatment, the grain yield of the Kabuli chickpea type was positively influenced by four major traits including HI, SDM, GN, and GW as a direct effect of $0.214,0.561,0.264$, and 0.139 , respectively. In this Kabuli type, very low residual variations of $3.1 \%$ and $1.11 \%$ were detected in grain yield variances at the full and limited irrigation treatments, respectively. The 
grain yield of Desi chickpea genotypes was contributed positively by effects of $0.247,0.294,0.736$, and 0.637 belonged to $\mathrm{HI}, \mathrm{SDM}, \mathrm{GN}$, and GW, respectively, under full-irrigated treatment. The GW and MT shared negative contributions with grain yield by -0.155 and -0.205 respectively, however, SDM, HI, GN, and FM yielded positive effects on grain yield of Desi chickpeas harvested in water-limited condition. The residual variations of $3.9 \%$ and $2.8 \%$ were observed for grain yield variances of Desi chickpea genotypes in full and limited irrigation treatments, respectively. The most influence on grain yield was detected for SDM at all irrigation treatments expect Desi type in full irrigation ones, which was distinguished by GN. The biggest influence on $\mathrm{HI}$ was recorded through GN in Kabuli chickpeas as a positive effect, while Desi type showed that SDM has the biggest influence on $\mathrm{HI}$ as a negative effect, both of them in all irrigation treatments. GN, in addition, affected GW in water-limited condition with a decreasing effect, while did show not contribution to GW in full-irrigated Kabuli chickpeas. GN affected the GW negatively in Desi chickpeas at both limited and full irrigation conditions. Among measured phenological characters, only FM and MT had a direct contribution to grain yield just in Desi chickpeas at the limited water condition.

\section{Discussion}

Genetic variation within cultivated chickpea genotypes can be exploited to improve drought tolerance in future varieties. On the other hand, a dependency of selection for drought-tolerant genotypes on grain yield under drought stress, which is controlled by many genes as well as the variability in drought stress patterns has created challenges in efforts to improvement chickpea performance and understanding the genetic control of drought stress tolerance. In addition, unpredictable precipitations in semi-arid lands persuade plants to suffer water restrictions in a situation, which has been not foreseen. Hence, knowledge of plant survival responses in the water limitations primarily and the next use of these learning to decrease destructive effects of the lack of water especially in reproductive stages are important issues in achieving acceptable performance. Natural selection favors plant survival mechanisms under severe stress, whereas plant breeding has emphasized selection for increased cost-effective yield (Blum, 2011). Thudi et al. (2017) believe that the complex nature of environmental stresses such as drought as well as a narrow genetic base in the cultivated gene-pool of chickpea are the major limiting factors, which kept its grain yield lesser than 1 ton per hectare.

Phenology of plants has an immense influence on productivity and stability (Upadhyaya et al, 2011), therefore, appropriate time of flowering is a major component of crop adaptation, particularly in environments where the growing season is restricted by terminal drought (Subbarao et al, 1995). FT at both Kabuli and Desi chickpeas grown under water-limited conditions showed negative effects on FM, indirectly causing reduce in GW and GY, respectively (Figures 4 and 6). The greater PT led to increased GN as well as a decrease in GW at full-irrigated Desi chickpeas, while caused reduce in GN at water-limited conditions (Figures 5 and 6). However, PT of Kabuli chickpea genotypes did have not a direct effect on either GY or its components. The decrease of FM of Kabuli type was fewer (3 days) compared with Desi type (7 days) in stressed plants (Table 2), which was in agreement with Nayyar et al (2006). Various 
scenarios detected for FM among Desi and Kabuli chickpeas could be emphasized as of the important role of this trait in the chickpea plant's responses to drought. In full irrigation treatment, FM showed major positive contributions on SDM and GW in Kabuli type, while in Desi type had on HI. However, this effect was observed on $\mathrm{GW}$ and $\mathrm{HI}$ in Kabuli chickpeas and was detected for $\mathrm{GN}$ as well as directly on $\mathrm{GY}$ of Desi chickpeas at the limited water conditions. These results suggest that to be longer FM can protect chickpea plants to achieve more possible grain yield either on limited or without limited irrigation conditions. Considering to path analysis results, more MT in full-irrigated chickpeas did have not benefits for crop outlook. However, MT was decreased due to water stress by 9 and 7 days in Desi and Kabuli genotypes, respectively, validating previous reports (Pushpavalli et al, 2014, Urrea et al, 2010, Fang et al, 2010). Overall, it is concluded that begin shorter of FT followed by a longer FM could be an appropriate feature for chickpeas adapted to terminal drought conditions. Some studies also assumed early flowering and longer grain filling duration as important attributes of chickpea plants contributing to higher grain yield under terminal drought (Rehman et al, 2011, Bergeret et al, 2004, Ganjeali et al, 2011, Singh et al, 2005). Furthermore, It seems that selection exclusively based on the drought tolerance indices without surveying of other phenotypic attributes of plants leads to neglect of chickpea phenological traits, as the lack of significant correlations between phenological traits and the drought tolerance indices (Tables 3 and 4).

Grain yield of Kabuli chickpeas under water-limited treatment showed a positive correlation with GY in full irrigation treatment $(R 2=0.16)$, a significant relationship $(P \leq 0.05)$, which confirms yield potential could explain $16 \%$ of GY under terminal drought stress (Figure 1). This justification was approximately in accordance with chickpea potential (20\%) under salinity stress (Atieno et al, 2017). GY of Desi chickpeas under water-limited conditions, however, did not show a significant relationship with full irrigation condition, limiting suitable selection for drought-tolerant genotypes only in terminal drought conditions (Figure 1). The SI measured in this study detected that applied water stress has been equivalent of 50.70 and 44.05 units on the Kabuli and Desi chickpeas, respectively. Other studies mentioned vary ranges of reduced grain yield due to drought stress. GY has been decreased by $33 \%, 53 \%, 21 \%, 30$, and $66 \%$ reported by Fang et al (2010)., Urrea et al (2010)., Davies et al (1999)., Behboudian et al. (2001) and Nayyar et al. (2006), respectively, in Desi chickpeas and decreased by $24 \%, 80 \%$, and $15 \%$ reported by Leport et al. (1999), Nayyar et al. (2006) and Fang et al. (2010), respectively, in Kabuli chickpeas under terminal drought conditions. Nayyar et al. (2006) found that in terminal water stress, Kabuli chickpeas take care of the greater allocation of assimilates towards the maintenance of vegetative growth, unlike Desi type assigned greater amounts of assimilates towards developing grains. GMP index was selected as the most positive correlations with GY at the both limited and full-irrigated conditions, which was in agreement with Anwar et al. (2011) and El-Hendawy et al. (2017) in wheat as well as Khan et al. (2018), Jha et al. (2010) and Ganjeali et al. (2011) in chickpea. In addition, if GMP is considered as selection criteria, it could select genotypes having high GN, SDM, and GW of Kabuli type, and could select Desi chickpeas with high SDM and GN, which to be more tolerant to terminal drought stress, because of positive correlations (Tables 3 and 4). 
The inherent potential of Kabuli and Desi chickpeas can interpret $19 \%$ and $32 \%$ of SDM, respectively, produced at the terminal drought conditions (Figure 1). However, terminal water stress decreased SDM of Kabuli and Desi genotypes by $42.46 \%$ and $39.60 \%$, respectively. The decrease of SDM due to terminal drought stress has been reported by $28 \%$ in Kabuli as well as $23 \%$ and $34 \%$ in Desi chickpeas, respectively by other studies (Behboudian et al, 2001, Nayyar et al, 2006). Path analysis detected that $59 \%$ and $14 \%$ of GY variance has been justified by SDM in Kabuli and Desi chickpeas grown in full-irrigated condition, respectively. In water-limited conditions, SDM justified by $47 \%$ and $48 \%$ GY variance, respectively, in Kabuli and Desi types. In many studies, the most contribution of GY has been justified by SDM in optimal conditions (Saleem et al, 2002), under salinity stress (Zamani et al, 2017) and under rain-fed conditions (Arshad et al, 2004).

Terminal water stress decreased GW of Kabuli genotypes by $2.82 \%$, while, an increase of $2.22 \%$ was observed for GW in Desi chickpeas. Behboudian et al. (2001) found that GW in Desi chickpea had been not decreased under terminal water stress, which reason of this result was assumed as an accumulation of soluble sugars, amino acids, and proteins in chickpea grains. However, it seems that indirect selection for more drought-tolerant chickpeas could be performed through GW because of the greater correlation between drought and non-drought conditions than other attributes (Figure 1). The results of the variance component analysis also confirmed that GW has not been influenced by environmental factors (Table 7). Noor et al. (2003) found additive gene effects on GW of chickpea-based on high heritability with a high genetic advance in rain-fed conditions. Despite observations in Desi type, three indices including GMP, HM, and SNPI showed a highly positive significant correlation with GW in Kabuli type under drought stress condition. The GMP and HM also had a high correlation with GW at the non-stress conditions (Table 3). Being additive in effect of genes on GW is a sign of additively gene controlling, hence it could be used through simple and direct selection, which in turn, these indices could provide a promising direction while genetically improvement in Kabuli chickpea yield is followed under rain-fed conditions.

A relationship significant in 0.01 probability level confirmed that $33 \%$ of GN in Desi type yielded under water stress condition could be explained by the inherent potential of the crop (Figure 1). However, this relationship was not observed in the Kabuli genotypes. In Desi and Kabuli chickpea genotypes, terminal drought decreased up to $44.71 \%$ and up to $50.10 \%$ of GN, respectively (Table 2). GN of tested genotypes was influenced by water limitation more than other attributes (Table 7). STI and HM indices were the best indicators to select genotypes having more GN in Kabuli and Desi chickpeas, respectively (Tables 3 and 4). GN had a positive effect on GY at both chickpea types and conditions, which was in agreement with Pushpavalli et al. (2014). The HI of Kabuli and Desi chickpeas were reduced by $12.25 \%$ and $9.74 \%$, respectively, due to the terminal water stress (Table 2). Nayyar et al. (2006) also found greater decreasing of $\mathrm{HI}$ in Kabuli than Desi chickpeas under terminal drought and stated that presence of relatively higher vegetative dry matter at maturity in Kabuli than Desi genotypes may be a sign of its lower ability to remobilize the assimilates towards developing pods and grains. Plants with higher HI shown better partitioning of photosynthetic assimilate to grain development under water stress conditions. This ability should help the crop in improving its stability of performance under different climatic conditions compared to selection based solely on grain yield (Rehman et al, 2011). The research conducted on 
Kabuli chickpea showed that precipitation in the last two months of plant growth time has been caused an increasing $\mathrm{HI}$ from $0.5 \%$ to $0.34 \%$ as compared to lack of rain at the same time in sensitive genotype (ILC 3279), whereas non-significant difference was observed in tolerant genotype (ILC 588) (Rehman et al, 2011). The most contribution of $\mathrm{HI}$ belonged to GN in both chickpea types either in stress and non-stress conditions (Figures 3, 4, 5, and 6). Plants that combine high values of canopy biomass with greater mobilization of photosynthates to grains have been assumed as drought tolerant. Although $\mathrm{HI}$ in Kabuli genotypes did not affect directly by SDM, in Desi genotypes the SDM had a positive effect on $\mathrm{HI}$, which could be evidence of the photosynthates mobilization to grains. On the other, at both chickpea types, there was not any correlation between $\mathrm{HI}$ and the drought tolerance indices in water-limited conditions. However, some of the indices such as GMP, MP, ATI, TOL, and K1STI in Kabuli type as well as TOL and ATI in Desi showed positive and significant correlations with $\mathrm{HI}$ under full irrigation conditions. According to these results, it seems that the improvement of $\mathrm{HI}$ in chickpeas grown under optimal water condition is a straighter approach than selection under terminal water stress.

Considering non-significant estimated genotypic variance of GY in the combined analysis, which could be due to the complexity of involved mechanisms, it seems that indirect selection through each of GW, SDM, and GN could result to more repeatable outcomes (Table 7). Breeding for drought tolerance by selection based on GY solely is difficult, because low heritability of GY under drought conditions, which is due to small genotypic variance or large genotype by environment interaction variances (Ludlow and Muchow, 1990). Environmental factors highly influenced the genetic structure and phenotypic expression of a quantitative trait such as GY, thus genotype by environment interactions is a major barrier for understanding that of inheritance (Breese, 1969). The contribution of genotypic variances as equivalent to the heritability of GY, GN, GW, and SDM in Desi chickpeas was greater in full irrigation condition than water-limited condition. Hence, it could be said that selection without terminal drought conditions will lead to more repeatable results than selection under terminal water stress. In Kabuli chickpeas, however, the greater genotypic variances were detected for GN and SDM in full irrigation condition and for GY and GW were observed under limited-water condition. Therefore, according to the objectives of the selection, doing this selection under conditions with greater genotypic variances dedicated to each trait is better. Hence, as Desi chickpea genotypes 8, 10, 47, and 321 showed significant positive predicted genotypic effects under optimal conditions for the selection, involving these genotypes in multi-parent recombination crosses could be resulting in increased efficient performance. In Kabuli chickpeas, the genotypes $101,21,15,25$, and 166 were detected as those of better ones with significant positive predicted genotypic effects.

According to Yan and Kang (2002), an ideal genotype should have a high yield mean among stress and non-stress environments as well as show high stable performance. Rad et al. (2013) stated that the ideal genotype could be found in the center of the concentric circles of AEC method analysis. As AEC abscissa direction towards a more stable grain yield, as shown in Figure 2, the ideal chickpea genotypes have been presented closely to the location of a limited water environment as well as the average environment. As a result, which found consistent with Golabadi et al. (2006) in durum wheat concluded that for high stable grain yield, selection of chickpea in moister-stress environments as well as based on the average of 
drought stress and non-drought stress conditions could be more advantageous compared with indirect selection only at the non-drought stress conditions.

\section{Conclusion}

Results of this study showed that tested chickpea genotypes responded differently under different water treatments, suggesting the importance of assessment of genotypes under these conditions in order to identify the best genotype make up for each particular condition. As water stress severity was applied equally, therefore it was thought to be more serious in genotypes with a greater life cycle. However, it seems that chickpea plants have been adapted to the terminal drought stress, which could be due to the same time of vegetative growth with filling pods and transfer capability of photosynthesis assimilates towards more grain yield in tolerant genotypes. It seems to change in plant phenology due to the terminal drought stress more affected GN and GW in Desi and Kabuli chickpeas, respectively. These differences could be clear points for the leadership of breeding programs towards more adaptation of both Desi and Kabuli chickpea types to terminal water stress, respectively. Moderate to a high proportion of $\mathrm{G} \times \mathrm{E}$ effects were observed in combined analysis for GY, GN, and SDM compared to genotypic effects, suggesting that $\mathrm{G} \times \mathrm{E}$ effects played a greater role than genotypic effects. The ideal genotype of Kabuli type i.e. genotype 25 had greater GY as well as SDM in water-limited condition, while genotype 321 as ideal Desi genotype showed acceptable GY and SDM, but could compensate with higher GN.

\section{Abbreviations}

GY: grain yield, GW: 100-grain weight, GN: number of grains, HI: harvest index, FT: days to flowering, PT: days to podding, FM: days from flowering to maturity, MT: days to maturity, SI: stress intensity,

\section{Declarations}

\section{Acknowledgement}

We do acknowledge the Pulses Center of Excellence for supporting of this research work along with Gene Bank of Department of Agronomy and Plant Breeding, College of Agriculture and Natural Resources, University of Tehran for providing chickpea seeds for carrying out this research work.

\section{Authors' contributions}

AT: Performed field trial, analyzed the datasets, and wrote the manuscript.

JS: Assistance in field operation, data analysis and writing the manuscript.

\section{Funding}

This work was supported by the Pulses Center of Excellence along with Gene Bank of Department of Agronomy and Plant Breeding, College of Agriculture and Natural Resources, University of Tehran for 
providing chickpea seeds for carrying out this research work. The funding body was involved in the material creation, designing the study, data analysis and writing the manuscript.

\section{Availability data and materials}

The datasets generated and analyzed during the current study could be available on request.

\section{Declarations:}

We declare that we approve the following and also I acknowledged Pulses Center of Excellence for supporting of this research work along with Gene Bank of Department of Agronomy and Plant Breeding, College of Agriculture and Natural Resources, University of Tehran for providing chickpea seeds for carrying out this research work. I need to clarify that second person helped me to carry out this research work.

\section{Ethics approval and consent to participate}

Not applicable

\section{Consent for publication}

Not applicable

\section{Competing interests}

The authors declare that they have no competing interests. The funding body was involved in the material creation, designing the study, data analysis and writing the manuscript.

\section{Author details}

Department of Agronomy and Plant Breeding, Faculty of Agricultural Sciences and Engineering, College of Agriculture and Natural Resources, University of Tehran

\section{References}

1 Anwar J, Subhani GM, Hussain MA, Ahmad J, Hussain M and Munir M. Drought tolerance indices and their correlation with yield in exotic wheat genotypes. Pak J Bot, 2011. 43: 1527-1530.

2 Arshad M, Bakhsh A and Ghafoor A. Path coefficient analysis in chickpea (Cicer arietinum L.) under rain-fed conditions. Pak J Bot, 2004. 36: 75-82.

3 Atieno J, Li Y, Langridge P, Dowling K, Brien C, Berger B, Varshney RK and Sutton T. Exploring genetic variation for salinity tolerance in chickpea using image-based phenotyping. Sci Rep, 2017. 7: 1300. 
4 Behboudian MH, Ma Q, Turner NC and Palta JA. Reactions of chickpea to water stress: yield and seed composition. J Sci Food Agric, 2001. 81: 1288-1291.

5 Berger JD, Turner NC, Siddique KH, Knights EJ, Brinsmead RB, Mock I, Edmondson C and Khan TN. Genotype by environment studies across Australia reveal the importance of phenology for chickpea (Cicer arietinum L.) improvement. Aust J Agric Res, 2004. 55: 1071-1084.

6 Blum A, Plant water relations, plant stress and plant production. In: Plant breeding for water-limited environments, New York, NY, Springer, 2011. 11-52.

7 Breese ED. The measurement and significance of genotype-environment interactions in grasses. Heredity, 1969. 21: 27-47.

8 Davies SL, Turner NC, Siddique KH, Leport L and Plummer JA. Seed growth of Desi and Kabuli chickpea (Cicer arietinum L.) in a short-season Mediterranean-type environment. Aust J Exp Agr, 1999. 39: 181-188.

9 El-Hendawy S, Al-Suhaibani N, Salem AEA, Rehman SU and Schmidhalter U. Spectral reflectance indices as a rapid and non-destructive phenotyping tool for estimating different morpho-physiological traits of contrasting spring wheat germplasms under arid conditions. Turk J Agric For, 2015. 39: 572-587.

10 El-Hendawy SE, Hassan WM, Al-Suhaibani NA and Schmidhalter U. Spectral assessment of drought tolerance indices and grain yield in advanced spring wheat lines grown under full and limited water irrigation. Agr Water Manage, 2017. 182: 1-12.

11 Fang X, Turner NC, Yan G, Li F and Siddique KHM. Flower numbers, pod production, pollen viability, and pistil function are reduced and flower and pod abortion increased in chickpea (Cicer arietinum L.) under terminal drought. J Exp Bot, 2010. 61: 335-345.

12 Ganjeali A, Porsa H and Bagheri A. Assessment of Iranian chickpea (Cicer arietinum L.) germplasms for drought tolerance. Agr Water Manage, 2011. 98:1477-1484.

13 Golabadi M, Arzani A and Maibody SM. Assessment of drought tolerance in segregating populations in durum wheat. Afr J Agr Res, 2006. 1: 162-17.

14 Hassan M, Atta BM, Shah TM, Haq MA, Syed H and Alam SS. Correlation and path coefficient studies in induced mutants of chickpea (Cicer arietinum L.). Pak J Bot, 2005. 37: 293-298.

15 Jha UC, Basu P, Shil S and Singh NP. Evaluation of drought tolerance selection indices in chickpea genotypes. Int J Bio-Res Stress Manage, 2016. 7: 1244-1248.

16 Jukanti AK, Gaur PM, Gowda CL and Chibbar RN. Chickpea: nutritional properties and its benefits. Brit J Nutr, 2012. 108: 11-26. 
17 Khan H, Gul R, Khan NU, Naz R, Shah S, Asim N and Latif A. Role of selection indices in ascertaining high yielding drought stress tolerant chickpea (Cicer arietinum L.). J Anim Plan Sci, 2018. 28: $146-154$.

18 Leport L, Turner NC, French RJ, Barr MD, Duda R, Davies SL, Tennant D and Siddique KH. Physiological responses of chickpea genotypes to terminal drought in a Mediterranean-type environment. Eur $J$ Agron, 1999. 11: 279-291.

19 Ludlow MM and Muchow RC. A critical evaluation of traits for improving crop yields in water-limited environments. Adv Agron, 1990. 43: 107-153.

20 Nayyar H, Kaur S, Singh S and Upadhyaya HD. Differential sensitivity of Desi (small-seeded) and Kabuli (large-seeded) chickpea genotypes to water stress during seed filling: effects on accumulation of seed reserves and yield. J Sci Food Agric, 2006. 86: 2076-2082.

21 Noor F, Ashaf M, Ghafoor A. Path analysis and relationship among quantitative traits in chickpea (Cicer arietinum L.). Pak J Biol Sci, 2003. 6: 551-555.

22 Pushpavalli R, Zaman-Allah M, Turner NC, Baddam R, Rao MV and Vadez V. Higher flower and seed number leads to higher yield under water stress conditions imposed during reproduction in chickpea. Funct Plant Biol, 2014.42: 162-174.

23 Rad MN, Kadir MA, Rafii MY, Jaafar HZ, Naghavi MR and Ahmadi F. Genotype environment interaction by AMMI and GGE biplot analysis in three consecutive generations of wheat (Triticum aestivum) under normal and drought stress conditions. Aust J Crop Sci, 2013. 7: 956-961.

24 Rao CR. Estimation of variance and covariance components-MINQUE theory. J Multivariate Anal, 1971. 1: 257-275.

25 Rao IM. Advances in improving adaptation of common bean and Brachiaria forage grasses to abiotic stress in the tropics. In: Handbook of plant and crop physiology, Pessarakli M (ed), Boca Raton, CRC Press, 2014. 876-919.

26 Rehman AU, Malhotra RS, Bett K, Taran B, Bueckert R and Warkentin TD. Mapping QTL associated with traits affecting grain yield in chickpea (Cicer arietinum L.) under terminal drought stress. Crop Sci, 2011. 51: 450-463.

27 Saleem MU, Tahir MH, Kabir RE, Javid MU and Shahzad KA. Interrelationships and path analysis of yield attributes in chickpea (Cicer arietinum L.). Int J Agric Boil, 2002. 4: 404-406.

28 Schneider SH, Semenov S, Patwardhan A, Burton I, Magadza CHD, Oppenheimer M, Pittock AB, Rahman A, Smith JB, Suarez A and Yamin F. Assessing key vulnerabilities and the risk from climate change. In: Climate change, Parry ML, Canziani OF, Palutikof JP, van der Linden PJ and Hanson CE (eds). Cambridge: Cambridge University Press, 2007. 779-810. 
29 Singh G, Sekhon HS and Kolar JS. Pulses. Agrotech Publishing Academy, 2005. Udaipur, India.

Subbarao GV, Johansen C, Slinkard AE, Nageswara Rao RC, Saxena NP, Chauhan YS and Lawn RJ. Strategies for improving drought resistance in grain legumes. Crit Rev Plant Sci, 1995. 14: 469-523.

30 Taleei A and Shaabani J. Yield potential analysis of Kabuli chickpea genotypes at the limited water conditions along with surveying of the drought tolerance indices. Int J Biosci Biotec. 2017a. 9: 11-24.

31 Taleei A and Shaabani J. Yield potential screening of Desi chickpea genotypes in water stress conditions. Int J Biosci Biotec. 2017b. 9: 89-102.

32 Thudi M, Gaur PM, Krishnamurthy L, Mir RR, Kudapa H, Fikre A, Kimurto P, Tripathi S, Soren KR, Mulwa $\mathrm{R}$ and Bharadwaj C. Genomics-assisted breeding for drought tolerance in chickpea. Funct Plant Biol, 2014. 41: 1178-1190.

33 Thudi M, Roorkiwal M, Kudapa H, Chaturvedi SK, Singh NP and Varshney RK. An overview of chickpea research: from discovery to delivery. Pulse India, 2017. 2: 22-25.

34 Toker C, Lluch C, Tejera NA, Serraj R, and Siddique KHM. Abiotic stresses. In: Chickpea breeding and management, Yadav SS, Redden R, Chen W, and Sharma B (eds). Wallingford, CABI, 2007. 474-496.

35 Upadhyaya HD, Dronavalli N, Gowda CL and Singh S. Identification and evaluation of chickpea germplasm for tolerance to heat stress. Crop Sci, 2011. 51: 2079-2094.

36 Urrea CA, Harveson RM, Koehler AE, Burgener $\mathrm{P}$ and Baltensperger DD. Evaluating the agronomic potential of chickpea germplasm for western Nebraska. Agron J, 2010. 102:1179-1185.

37 Wang JY, Xiong YC, Li FM, Siddique KH and Turner NC. Effects of drought stress on morphophysiological traits, biochemical characteristics, yield, and yield components in different ploidy wheat: A meta-analysis. Adv Agron. 2017. 143: 139-173.

38 Wold $\mathrm{H}$, Soft modeling. In: the basic Design and some extensions, in systems under indirect observations, Jöreskog G and Wold H (eds). Amsterdam, North-Holland, 1982. 1-54.

$39 \mathrm{Wu}$ J. GenMod: an R package for various agricultural data analyses. In: ASA, CSSA, and SSSA. International Annual Meetings, Cincinnati, Ohio. 2012.

40 Yadav SS, Kumar J, Yadav SK, Singh S, Yadav VS, Turner NC and Redden R. Evaluation of Helicoverpa and drought resistance in Desi and Kabuli chickpea. Plant Genet Resour, 2006. 4: 198-203.

41 Yan W and Kang MS. GGE biplot analysis: A graphical tool for breeders, geneticists, and agronomists. Boca Raton, CRC press, 2002. 288pp.

42 Yan W, Hunt LA, Sheng Q and Szlavnics Z. Cultivar evaluation and mega-environment investigation based on the GGE biplot. Crop Sci, 2000. 40: 597-605. 
43 Yan W. GGE biplot-a windows application for graphical analysis of multi-environment trial data and other types of two-way data. Agron J, 2001. 93: 1111-1118.

44 Zamani GR, Shaabani J and Izanloo A. Silicon effects on the growth and yield of chickpea under salinity stress. Int J Agric Biol, 2017. 19: 1475-1482.

45 Zhu J. Estimation of genetic variance components in the general mixed model (Doctoral dissertation, North Carolina State University. 1989.

46 Zhu J. Methods of predicting genotype value and heterosis for offspring of hybrids. Int J Biomath, 1993. 8: 32-44.

\section{Tables}

Due to technical limitations, the tables are only available as a download in the supplemental files section.

\section{Figures}



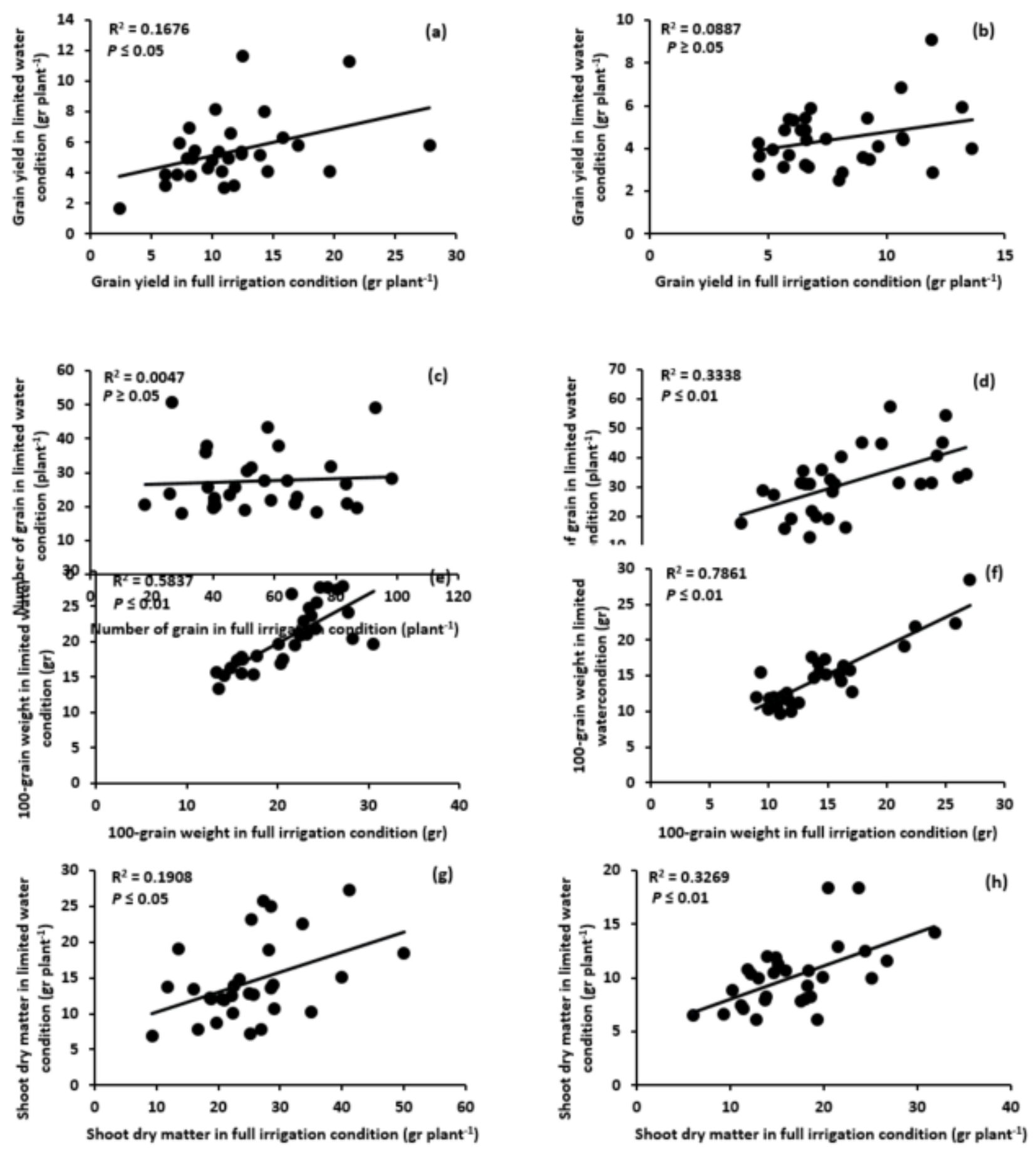

Figure 1

Association between grain yield (gr plant-1), number of grain (plant-1), 100-grain weight (gr), and shoot dry matter (gr plant-1) in full irrigation and limited water conditions; as a, c, e, and g letters belongs to Kabuli and b, d, f, and $\mathrm{h}$ letters are belong to Desi chickpea genotypes. 


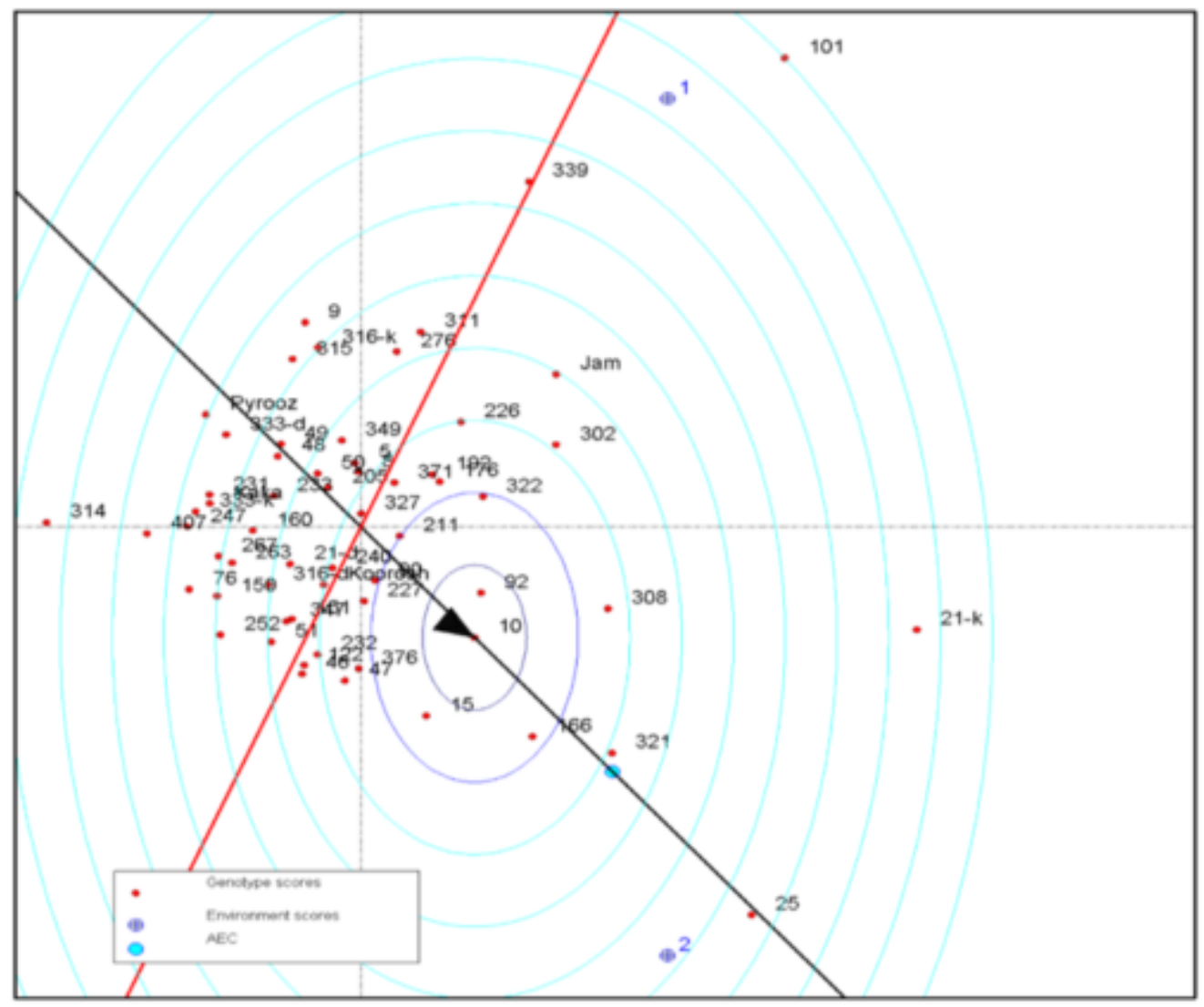

Figure 2

GGE biplot analysis based on genotype-focused scaling for comparison all Kabuli and Desi chickpea genotypes with the ideal genotype. Black and blue numbers stand for genotypes and environments, respectively. 


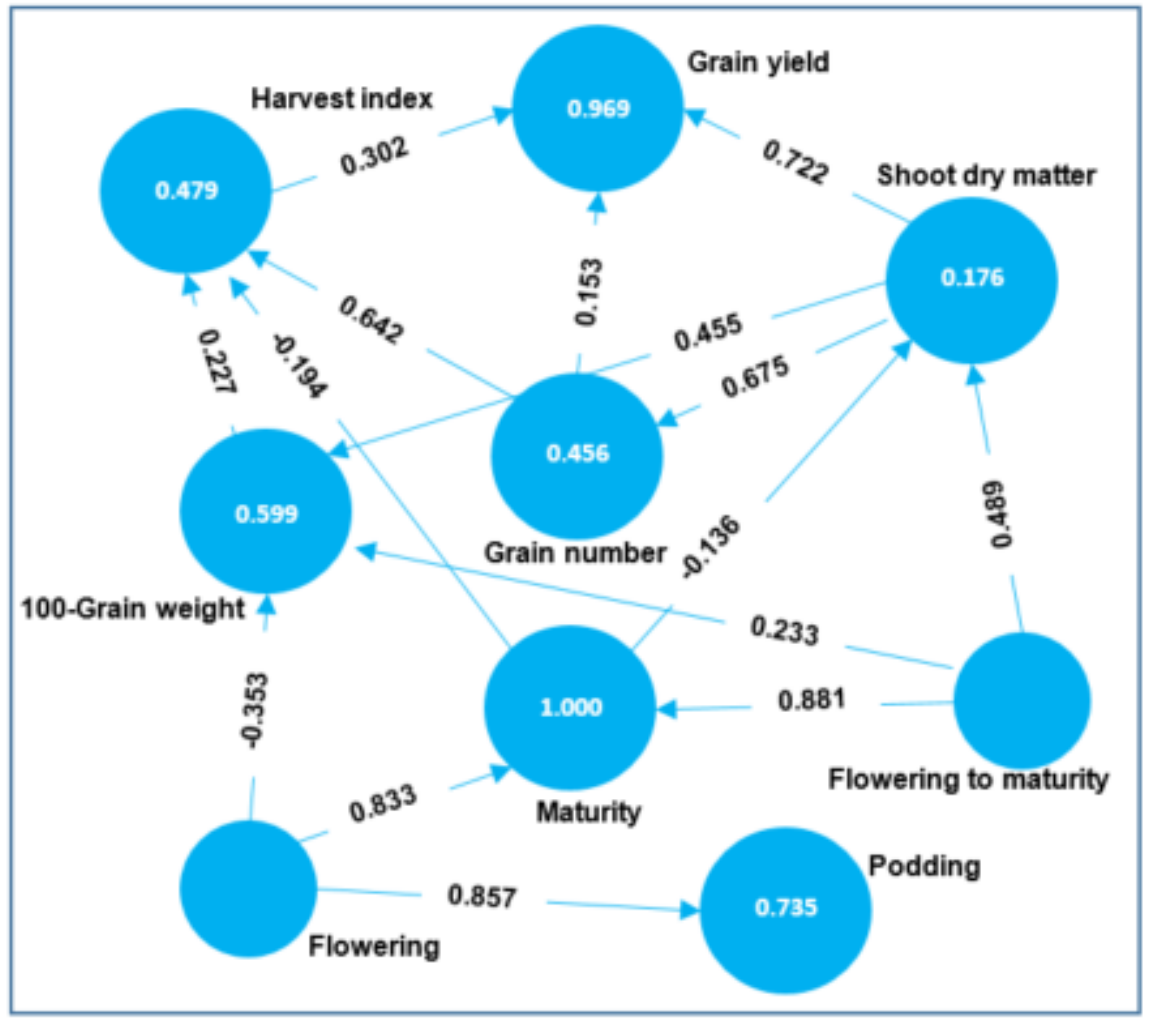

\section{Figure 3}

Path analysis diagram of grain yield (dependent variable) and other studied traits (independent variables). Path analysis derived from structural equation modeling using Partial Least Squares Algorithm method developed by Wold22 to determine complex relationship existing between grain yield and its related traits in Kabuli chickpea genotypes at the full irrigation condition. Path coefficients indicated with values on the arrows show a direct effect between different yield-related traits. R squared coefficients are indicated by values in the circles. 


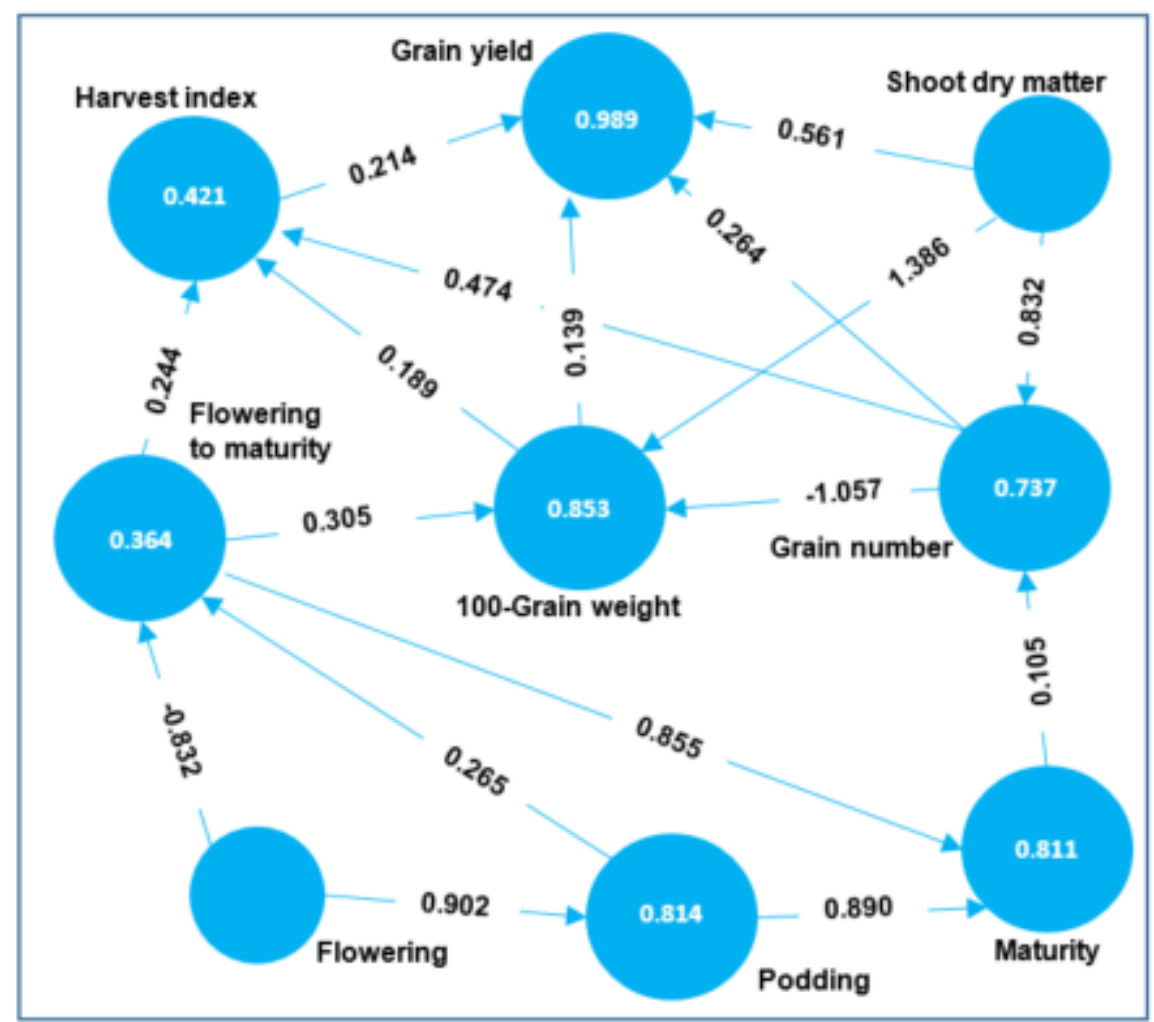

Figure 4

Path analysis diagram of grain yield (dependent variable) and other studied traits (independent variables). Path analysis derived from structural equation modeling using Partial Least Squares Algorithm method developed by Wold22 to determine complex relationship existing between grain yield and its related traits in Kabuli chickpea genotypes at the water-limited condition. Path coefficients indicated with values on the arrows show a direct effect between different yield-related traits. R squared coefficients are indicated by values in the circles. 


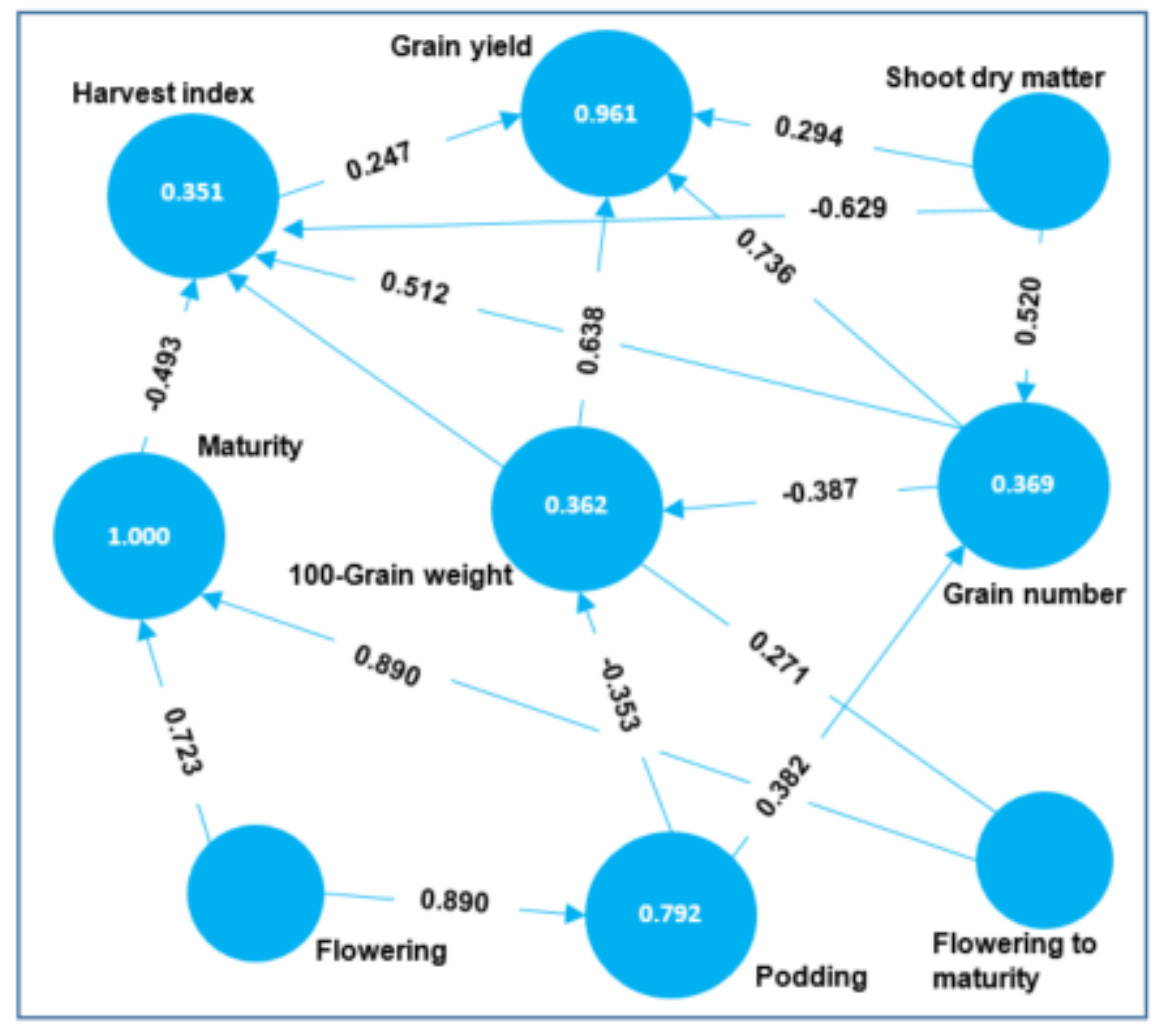

\section{Figure 5}

Path analysis diagram of grain yield (dependent variable) and other studied traits (independent variables). Path analysis derived from structural equation modeling using the Partial Least Squares Algorithm method developed by Wold22 to determine the complex relationship existing between grain yield and its related traits in Desi chickpea genotypes at the full irrigation condition. Path coefficients indicated with values on the arrows show a direct effect between different yield-related traits. $R$ squared coefficients are indicated by values in the circles. 


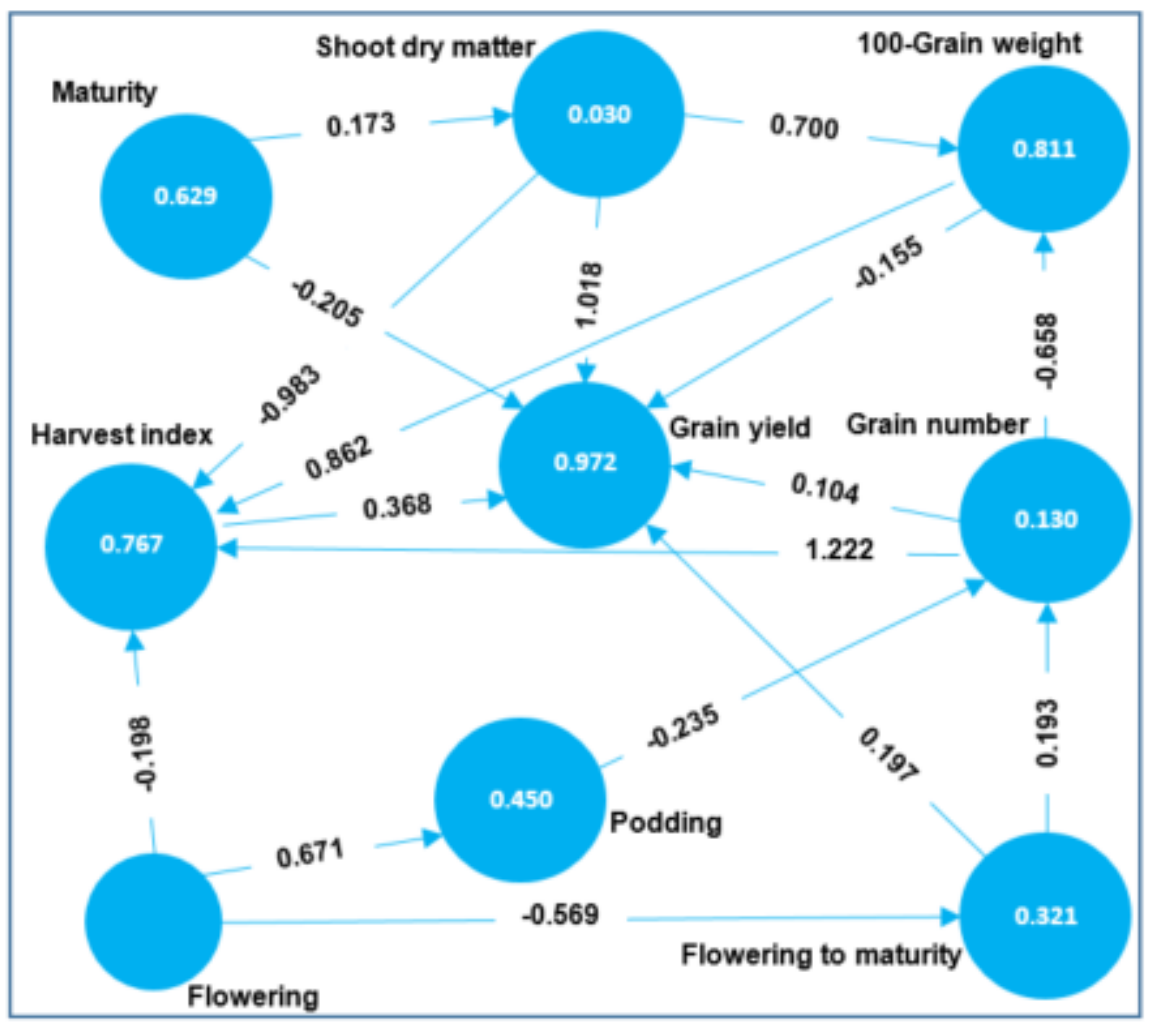

Figure 6

Path analysis diagram of grain yield (dependent variable) and other studied traits (independent variables). Path analysis derived from structural equation modeling using Partial Least Squares Algorithm method developed by Wold22 to determine complex relationship existing between grain yield and its related traits in Desi chickpea genotypes at the water-limited condition. Path coefficients indicated with values on the arrows show a direct effect between different yield-related traits. R squared coefficients are indicated by values in the circles

\section{Supplementary Files}

This is a list of supplementary files associated with this preprint. Click to download.

- table.pdf 